\title{
Rheology Based Design of Shear Thickening Fluid for Soft Body Armor Applications
}

\author{
Balasubrahmanya Harish Manukonda', Victor Avisek Chatterjee', Sanjeev Kumar Verma², \\ Debarati Bhattacharjee², Ipsita Biswas², Swati Neogi ${ }^{1 *}$
}

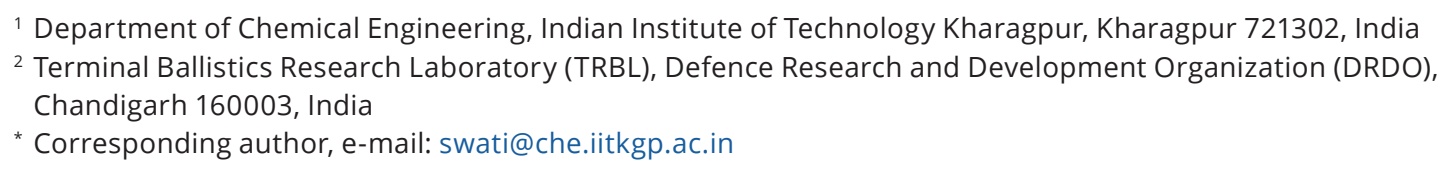

Received: 20 December 2018, Accepted: 18 March 2019, Published online: 03 April 2019

\begin{abstract}
The ballistic resistance of high-strength fabrics improves upon impregnation with Shear Thickening Fluids (STFs). The performance of such STF treated fabrics depends on the rheological properties of the STF which in turn are governed by the physicochemical properties of the STF. The present study utilizes rheological characterization of shear thickening silica-polyethylene glycol dispersions (of different material configurations in terms of packing fraction, particle size and continuous phase viscosity) to assess their performance and obtain the best STF material configuration for ballistic body armor applications based on the design criteria proposed herein. The ballistic performance assessment results showed that the STFs with high packing fractions which thicken discontinuously, are highly effective compared to the continuously shear thickening fluids. Furthermore, the use of smaller particle size dispersed phase in the STF formulation was determined to be economical. Also, the use of lower molecular weight dispersion medium was suggested as it allows for a broader working temperature range of the STF. Additionally, the technological issues associated with the development and the practical application of STF-Armor were addressed.
\end{abstract}

Keywords

shear thickening fluid, colloidal silica, rheological characterization, ballistic performance, design

\section{Introduction}

Shear thickening fluids (STFs) are a class of non-Newtonian fluids, often colloidal dispersions, in which apparent viscosity increases when the shear rate is above a critical value and this flow behavior is dramatic at high particle concentrations called, Discontinuous Shear Thickening (DST). This phenomenon causes the STF to translate from a liquid-like state (at lower shear rates) to a solid-like state (at higher shear rates) due to a rapid increase in apparent viscosity. This rate dependent mechanical property of STFs is exploited in various practical applications for energy dissipation: body armor for ballistic and stab protection $[1,2]$, damping and control devices for structures and automotives [3-6], fragment containment in aircraft engines [7] and orbital debris shielding in spacecrafts $[8,9]$.

Currently soft body armor research is focusing on development of light weight, flexible and comfortable armors with improved ballistic impact resistance [10]. The conventional ballistic vest is composed of multiple layers of fabric made of high tenacity fibres characterized by high energy absorption [11]. But to meet the protection requirements for typical ballistic threats, approximately 20-50 layers of this high-strength fabrics are required, resulting in bulky and stiff armor limited by its comfort and consequently restricting its application primarily to torso protection [1]. However, a significant number of battlefield casualties would actually happen due to injuries inflicted on body extremities such as hands, arms, neck, and legs [12]. Studies have reported and shown that STF impregnated Kevlar fabric is both flexible and thinner with ballistic performance comparable to that of the corresponding neat Kevlar fabric of equal weight [1]. STFs possess low apparent viscosity under shear rates associated with normal motion of the wearer, offering little impediment to the flexure and deformability of the fabric but thickens rapidly at high shear rates of ballistic impact thereby enhancing the impact resistance of the impregnated fabric [12]. 
Thus, fewer layers of impregnated fabric are needed to attain the required ballistic performance resulting in a less bulky and more flexible armor. And the improvement in the ballistic protection behavior of the fabrics treated with STF may be due to the combined effect of some or all of the following mechanisms of energy absorption: (a) energy dissipation due to shear-thickening behavior, (b) increased yarn to yarn friction (yarn pull-out energy), (c) better coupling and load transfer between fibre to fibre and yarn to yarn [10]. The compact and pliable STF treated fabric composites offer maximum maneuverability without compromising safety so, are of potential use in extremity protection. And for the successful design of this composite armor, it is important to investigate the STF rheology and obtain the best STF formulation.

A typical viscosity-shear rate curve of a shear thickening fluid obtained from a conventional rheometer has three characteristic regions - slight shear thinning at low shear rates, followed by a sharp viscosity increase over a threshold shear rate value, and a subsequent pronounced shear thinning region at high shear rates which are represented by Zone I, Zone II and Zone III respectively in Fig. 1. The initial shear thinning and the following shear thickening can be explained by the order-disorder transition [13] and the hydroclustering [14], with the latter widely accepted as a more accurate and generalized micromechanical theory. Hydroclustering mechanism attributes the shear thickening phenomenon to the formation of flow-induced local transient

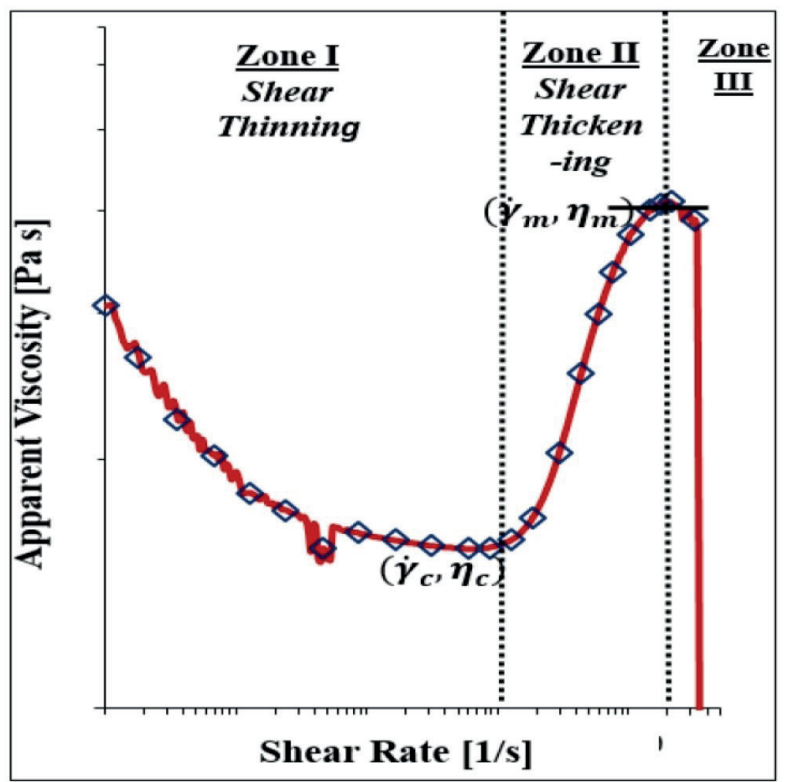

Fig. 1 Typical viscosity curve of a shear thickening fluid. Experimental data shown is corresponding to a suspension of $400 \mathrm{~nm}$ silica nanoparticle in polyethylene glycol at $60 \%(\mathrm{w} / \mathrm{w})$ and $25^{\circ} \mathrm{C}$. fluctuations in the particle concentration also referred to as hydroclusters. As the shear thickening develops, the instabilities formed on the free surface (liquid-air interface) eventually cause a portion of the sample between the rheometer plates to be ejected and this loss of sample reduces the torque required to maintain the shear rate, resulting in a decrease in the apparent viscosity (Zone III), but is not a representative of the true STF viscosity [9]. This region does not signify an actual decrease or drop in apparent viscosity, nor does it signify that the fluid has started behaving like a shear thinning fluid. As the shear rate increases after the Critical Shear Rate, the apparent viscosity of the STF increases and initiates instabilities on the free surface of the sample. As a result of these instabilities, a portion of the STF under test is forced out of the gap between the two plates thereby decreasing the gap length. This loss of sample results in a reduced torque required to maintain the shear rate, thereby resulting in the indication of a much lower apparent viscosity which is not indicative of the true apparent viscosity of the sample at that shear rate. Formation of hydrocluster with some other factors are considered to be a cause of the generation of such surface instabilities [9, 15]. Thus the shear rate at this point should not be confused as the maximum attainable apparent viscosity but rather the maximum measurable apparent viscosity.

The rheological characteristics that can be obtained directly from the viscosity curves are the zero shear viscosity $\left(\eta_{\mathrm{o}}\right)$ - refers to the asymptotic apparent viscosity value at very low shear rates, and critical shear rate $\left(\dot{\gamma}_{c}\right)$ - refers to the value of the shear rate at the onset of shear thickening.

The ballistic performance of STF is related to its shear thickening response, which can be further characterized by fitting the shear stress $(\tau)$ - shear rate $(\dot{\gamma})$ data of Zone II to the power law model (as in Eq. (1)) and estimating the flow consistency index $(k)$ and the flow behavior index $(n)$. The power law relation, though empirical, is known to represent the rheological properties of wide variety of non-Newtonian fluids better than other two constant models [16]. The dimensionless flow behavior index $(n)$ represents the degree of departure from Newtonian behavior, thus gives a measure of the strength or the severity of shear thickening.

$\tau=k(\dot{\gamma})^{n}$

STFs are generally concentrated stabilized dispersions of rigid submicron particles in a carrier fluid [5]. The rheological behavior is controlled by various factors which include physicochemical properties of dispersion like particle size, particle size distribution, particle shape, phase volume or 
packing fraction, medium viscosity etc. [17]. In this paper, the effects of packing fraction, particle size and medium viscosity of the STF on its rheological properties and its ballistic performance were presented. These results were correlated with the proposed design criteria to develop a general framework to formulate STF for ballistic applications. Also, the practical issues associated with the development and the application of STF-Armor were addressed.

\section{Materials and methods}

\subsection{Materials}

The materials used in this study include monodisperse spherical silica particles (of sizes $400 \mathrm{~nm}$ and $500 \mathrm{~nm}$ ), polyethylene glycol (of molecular weights $200 \mathrm{~g} / \mathrm{mole}$ and $400 \mathrm{~g} / \mathrm{mole}$, hereafter designated as PEG200 and PEG400 respectively) and absolute ethanol (99.9\%). Silica particles are known to give better shear thickening response as they are comparatively harder than other materials used for rheological studies. The PEG was chosen as the continuous phase due to its low volatility, thermal stability and non-toxicity. Also the matching refractive index values of silica and PEG enhances colloidal stability. Thus, SilicaPEG STF is an excellent choice for ballistic applications. Furthermore, monodisperse silica was used since widening the particle size distribution alleviates the severity of shear thickening [17]. The submicron silica particles of both the sizes were procured from TBRL (Chandigarh, India), PEG200 and PEG400 were supplied by Merck Specialities Pvt. Ltd. (Mumbai, India) and the ethanol was purchased from Honyon International, Inc. (Wujiang City, China).

\subsection{Synthesis}

The STFs were synthesized by a series of operations. Mixture containing known amount of silica and ethanol in a 1 to 3 ratio $(\mathrm{w} / \mathrm{w})$ was agitated by a magnetic stirrer (Remi, $5 \mathrm{MLH}, 5 \mathrm{~L}, 500 \mathrm{~W}$ ) at $300 \mathrm{RPM}$ for 30 minutes.
PEG was then added to this silica-ethanol mixture followed by 30 minutes of stirring at 300 RPM. The final mixture, maintained at low temperature by an ice bath, was ultrasonicated (Hielscher UP400S, $24 \mathrm{kHz}, 400 \mathrm{~W}$ ) for $4.5 \mathrm{hrs}$ at $50 \%$ amplitude. The sample was heated to and maintained at slightly above $80^{\circ} \mathrm{C}$ for sufficient time on a hot plate magnetic stirrer (Remi, $5 \mathrm{MLH}, 5 \mathrm{~L}, 500 \mathrm{~W}$ ) to eliminate the ethanol. Stirring was also carried out during this process to avoid localized heating. This hot STF was kept in a vacuum desiccator to prevent moisture absorption by the sample and to remove any ethanol left in the sample after the heating. Eight STFs with different combinations of particle loading, particle size and dispersing medium, were synthesized and their properties are summarized in Table 1. These combinations are referred to as STF/material configurations hereafter. While the particle loading refers to the percentage of silica by weight $(\mathrm{w} / \mathrm{w})$, the packing fraction $(\varphi)$ is the fractional volume $(\mathrm{v} / \mathrm{v})$ occupied by the silica particles in the synthesized Silica-PEG STF. The packing fraction was calculated using the Eq. (2),

$\varphi=\frac{\left(\frac{m}{\rho}\right)_{\mathrm{Si}}}{\left(\frac{m}{\rho}\right)_{\mathrm{Si}}+\left(\frac{m}{\rho}\right)_{\mathrm{PEG}}}$

where, $(\mathrm{m} / \rho)$ refers to the ratio of mass to the density, the subscript Si refers to silica and PEG to polyethylene glycol. The density of silica and PEG (PEG200 and PEG400) were taken as $2.4 \mathrm{~g} / \mathrm{cc}$ and $1.125 \mathrm{~g} / \mathrm{cc}$ respectively at $25^{\circ} \mathrm{C}$.

\subsection{Rheological Characterization}

Steady shear rheological measurements of the as-prepared STF samples were obtained using a controlled rate rheometer (Anton Paar, Physica MCR-301). All the tests were performed at $25^{\circ} \mathrm{C}$ using a parallel plate fixture with a plate diameter of $50 \mathrm{~mm}$ and a gap size of $0.5 \mathrm{~mm}$.

Table 1 Designations and material configurations of the synthesized STF samples.

\begin{tabular}{lcccc}
\hline Sample & Silica particle loading & Silica packing fraction & $\begin{array}{c}\text { Silica particle size } \\
{[\mathrm{nm}]}\end{array}$ & $\begin{array}{c}\text { PEG molecular weight } \\
{[\mathrm{g} / \mathrm{mole}]}\end{array}$ \\
\hline STF A & $60 \%$ & 0.4128 & 400 & 200 \\
STF B & $65 \%$ & 0.4654 & 400 & 200 \\
STF C & $67 \%$ & 0.4876 & 400 & 200 \\
STF D & $70 \%$ & 0.5224 & 400 & 200 \\
STF E & $65 \%$ & 0.4654 & 500 & 200 \\
STF F & $67 \%$ & 0.4876 & 500 & 200 \\
STF G & $70 \%$ & 0.5224 & 500 & 200 \\
STF H & $65 \%$ & 0.4654 & 500 & 400 \\
\hline
\end{tabular}


Each sample was pre-sheared at a shear rate of $0.1 \mathrm{~s}^{-1}$ for 5 minutes. During the test, the sample was sheared for 20 seconds at each shear rate to attain the steady shear condition. The rheological test on each sample was repeated thrice. The data points plotted in rheology curves of the samples represent the average value of the data points obtained from the three test runs.

\section{Results and discussion}

\subsection{Rheological Properties of the STFs}

The rheology curves of all the STFs, PEG200 and PEG400 are presented in Figs. 2-5. All the STFs showed typical concentrated hard sphere dispersion behavior of initial shear thinning followed by shear thickening and subsequent shear thinning (not shown here). The rheology of pure PEG200 and pure PEG400, carried out as control for the STFs showed slight shear thinning initially and reached newtonian behavior at moderate shear rates but no shear thickening which indicates that the shear thickening phenomenon is caused due to the presence of solid phase and cannot usually occur in pure materials. The apparent viscosity values of pure PEG200 and PEG400 were found to be $0.0438 \mathrm{~Pa}-\mathrm{S}$ and $0.0899 \mathrm{~Pa}-\mathrm{s}$ respectively. The zero shear viscosity $\left(\eta_{\mathrm{o}}\right)$ was obtained by extrapolating the low shear rate viscosities to zero shear rate. The critical shear rate $\left(\dot{\gamma}_{c}\right)$ was taken as the shear rate above which the apparent viscosity continuously increases in a viscosity-shear rate plot. The shear thickening data of each STF was fitted to the power law model (Eq. (1)) and the parameters were estimated. The rheological characteristics $\left(\eta_{\mathrm{o}}\right.$ and $\left.\dot{\gamma}_{\mathrm{c}}\right)$, the rheological parameters $(k$ and $n)$ and the correlation coefficients $\left(\mathrm{R}^{2}\right)$ of the fits are summarized in the Table 2 . The high values of $\mathrm{R}^{2}$ confirms the suitability of the power law model in describing the shear thickening behavior of the STFs.

\subsection{Effect of particle loading (packing fraction)}

The particle loading was varied between $60 \%$ and $70 \%$ for STFs with $400 \mathrm{~nm}$ silica and between $65 \%$ and $70 \%$ for STFs with $500 \mathrm{~nm}$ silica. PEG200 was used as a dispersed medium for all the STFs considered to study the effect of particle loading. At the loadings higher than $70 \%$ the fluidity of the synthesized STF was lost, so rheology could not be performed. The apparent viscosity of the STFs was found to be a strong function of particle loading more so at higher loadings. The zero shear viscosity $\left(\eta_{\mathrm{o}}\right)$ increased and the critical shear rate $\left(\dot{\gamma}_{c}\right)$ decreased with increase in loading. The flow behavior index $(n)$ increased (because of increase in severity of shear thickening) with loading where as an inverse trend was observed for flow consistency index $(k)$. The STFs of both particle sizes showed similar trends in their rheological properties with loading (Table 2). DST refers to discontinuous jump in shear stress or viscosity with increase in shear rate while a smooth increase in shear stress or viscosity with shear rate is referred to as Continuous Shear Thickening (CST). It can be observed from Fig. 2 and Fig. 3 that STF A, STF B, STF E and STF F thickened continuously where as STF C, STF D and STF G exhibited DST. The nature of shear thickening changed from continuous to discontinuous as the loading increased.

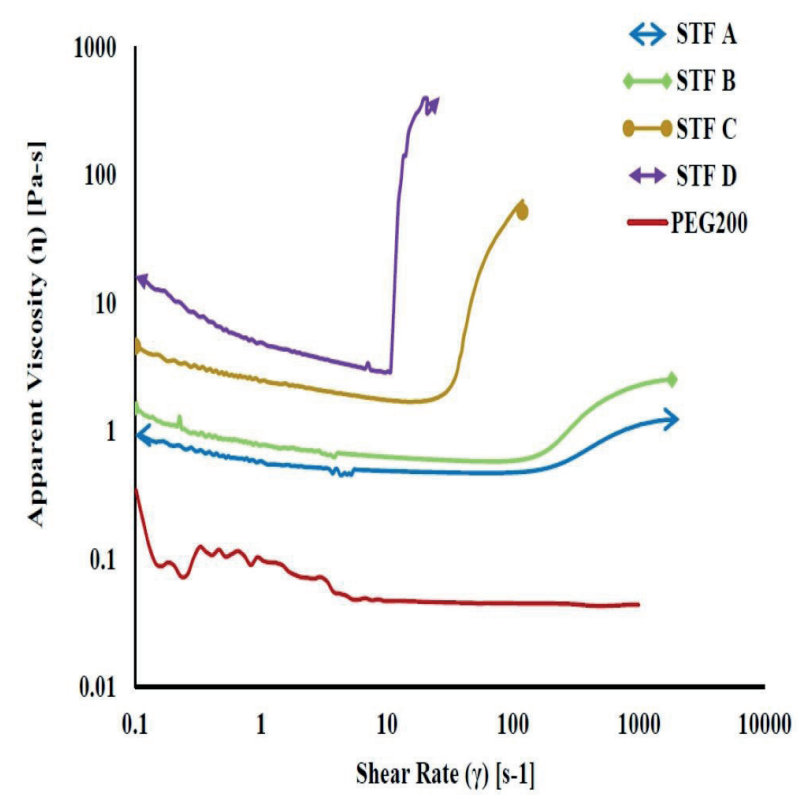

Fig. 2 Effect of particle loading on rheology of the STFs with $400 \mathrm{~nm}$ silica dispersed in PEG200.

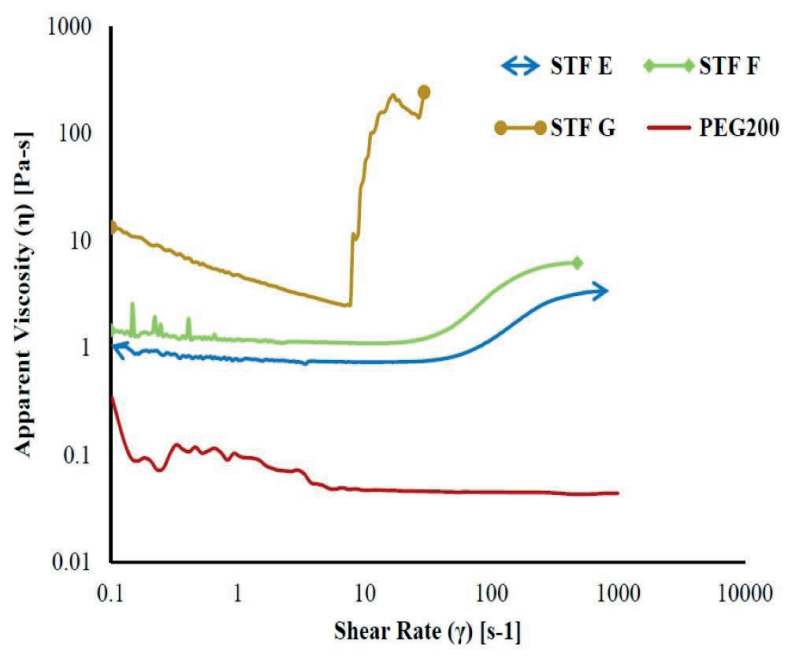

Fig. 3 Effect of particle loading on rheology of the STFs with $500 \mathrm{~nm}$ silica dispersed in PEG200. 
Table 2 Rheological properties of the synthesized STF samples.

\begin{tabular}{|c|c|c|c|c|c|c|c|c|}
\hline Rheological properties & STF A & STF B & STF C & STF D & STF E & STF F & STF G & STF H \\
\hline$\eta_{\mathrm{o}}[\mathrm{Pa}-\mathrm{s}]$ & 0.99 & 1.99 & 5.08 & 20.10 & 1.56 & 1.76 & 15.30 & 3.64 \\
\hline$\dot{\gamma}_{\mathrm{c}}\left[\mathrm{s}^{-1}\right]$ & 67.3 & 54.9 & 18.3 & 10.7 & 23.6 & 15.3 & 7.1 & 17.0 \\
\hline$k\left[\mathrm{~Pa}-\mathrm{s}^{\mathrm{n}}\right]$ & 0.03490 & 0.00700 & 0.00040 & 0.00005 & 0.04420 & 0.03660 & 0.00060 & 0.13380 \\
\hline$n$ & 1.5064 & 1.8860 & 3.6926 & 6.6410 & 1.7187 & 1.9651 & 5.8678 & 1.7273 \\
\hline $\mathrm{R}^{2}$ & 0.9995 & 0.9979 & 0.9933 & 0.9998 & 0.9989 & 0.9990 & 0.9706 & 0.9988 \\
\hline
\end{tabular}

\subsection{Effect of particle size and medium viscosity (molecular weight)}

The effects of particle size $(a)$ and medium viscosity $\left(\eta_{\mathrm{m}}\right)$ were studied at a moderate loading of $65 \%(\varphi=0.4654)$ so that the effects of particle loading are less dominant. The effect of particle size was studied by comparing the STF B $(65 \%, 400 \mathrm{~nm}, \mathrm{PEG} 200)$ and the STF E $(65 \%, 500 \mathrm{~nm}$, PEG200). It can be observed from Fig. 4, that the zero shear viscosity $\left(\eta_{0}\right)$ increased slightly and the critical shear rate $\left(\dot{\gamma}_{c}\right)$ decreased significantly with increase in particle size. While an order of magnitude increase was observed in the flow consistency index $(k)$, the flow behavior index (n) decreased with particle size increase (Table 2). The STF E $(65 \%, 500 \mathrm{~nm}$, PEG200) and STF H $(65 \%, 500 \mathrm{~nm}$, PEG400) are compared to study the effects of medium viscosity. The overall viscosity of a dispersion is almost always a direct function of the continuous phase viscosity [17], hence the $\eta_{\mathrm{o}}$ increased proportionally with medium viscosity (Fig. 5). The onset of shear thickening was shifted to lower shear rates with increase in medium viscosity. The severity of shear thickening is not improved so the value of $\mathrm{n}$ remained nearly constant with medium viscosity but the flow consistency index (k) improved (Table 2).

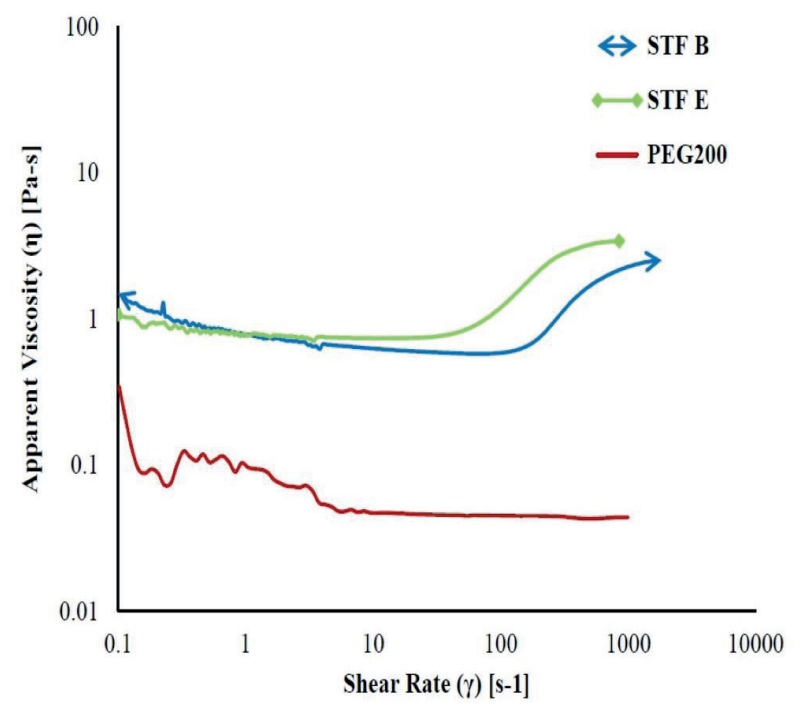

Fig. 4 Effect of particle size on the STF rheology.

\subsection{Ballistic performance assessment of the STFs}

The soft body armor is classified and designed based on the threat level of the ammunition. This threat level depends on the velocity of the bullet and on the bullet's design characteristics - size and weight of the bullet. The shear rate produced during ballistic impact, referred to as characteristic shear rate $\left(\dot{\gamma}_{\text {char }}\right)$ can be estimated based on the projectile velocity $(V)$ and the projectile diameter $(D)$ using Eq. (3) [1]. Table 3 shows the armor type, threat (in terms of velocity and diameter of the bullet according to National Institute of Justice (NIJ) Standard-0101.04 for the testing and performance of ballistic armor [18]) and the estimated impact shear rates.

$\dot{\gamma}_{\text {char }}=\frac{V}{D}$

The apparent viscosity-shear rate data obtained beyond Zone II (Fig. 1) is not a true material property of STF as a consequence, the magnitude of apparent viscosity increase that can be studied in a conventional rheometer is limited to shear rates (order of $10^{3} \mathrm{~s}^{-1}$, as seen in Figs. 2-5) significantly lower than the shear rates associated with ballistic impact (order of $10^{4}-10^{5} \mathrm{~s}^{-1}$, as seen in Table 3). However,

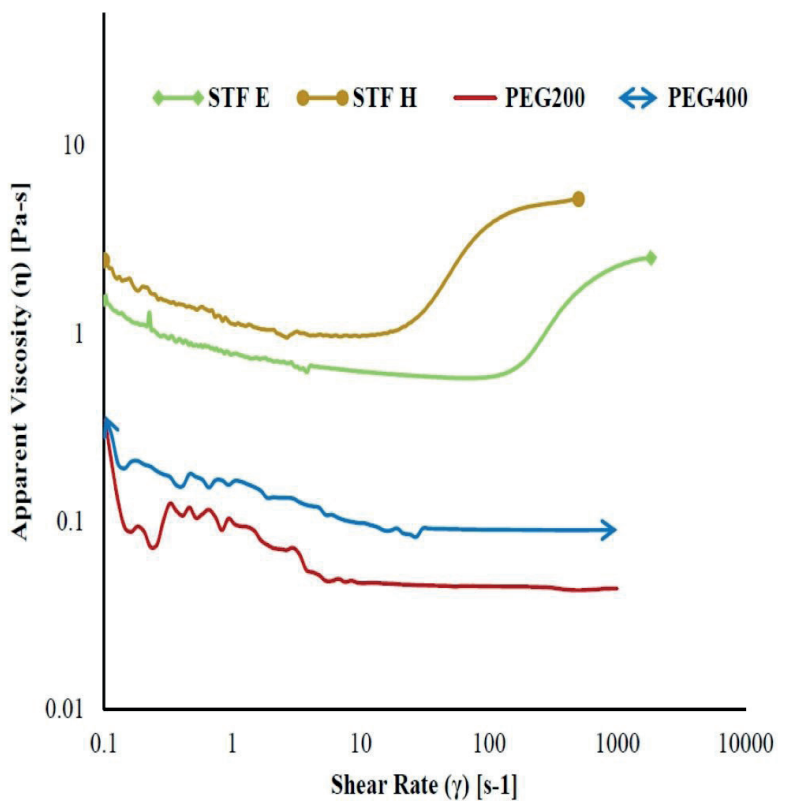

Fig. 5 Effect of medium viscosity (molecular weight) on the STF rheology. 
Table 3 Armor type, test bullet specifications and the corresponding characteristic shear rates.

\begin{tabular}{lccc}
\hline Armor type a $^{\text {a }}$ & $\begin{array}{c}\text { Bullet diameter } \\
{[\mathrm{mm}]}\end{array}$ & $\begin{array}{c}\text { aullet velocity } \\
{\left[\mathrm{mm}-\mathrm{s}^{-1}\right]}\end{array}$ & $\begin{array}{c}\text { Characteristic shear rate } \\
{\left[\mathrm{s}^{-1}\right]}\end{array}$ \\
\hline I & 5.60 & 329184 & 58782.86 \\
I & 9.70 & 321564 & 33150.93 \\
IIA & 9.00 & 341376 & 37930.67 \\
IIA & 10.20 & 321564 & 31525.88 \\
II & 9.00 & 367284 & 40809.33 \\
II & 9.10 & 435864 & 47897.14 \\
IIIA & 9.00 & 435864 & 48429.33 \\
IIIA & 11.20 & 435864 & 38916.43 \\
III & 7.62 & 847344 & 111200.00 \\
IV & 7.62 & 877824 & 115200.00 \\
\hline a As per NIJ Standard-0101.04 & & &
\end{tabular}

studies have always reported improvement in the ballistic performance of the conventional fabrics when impregnated with STF which suggests that the dispersion continues to thicken at shear rates beyond the typical shear thickening regime (Zone II, Fig. 1).

To measure the low-strain apparent viscosities, rheological measurement techniques were employed. But for the high strain rate characterization of STF, split Hopkinson pressure bar (SHPB) systems have been used by researchers such as Asija et al. [19, 21], Lim et al. [20] and Cao et al. [22]. Asija et al. [19] carried out the high strain-rate studies of spherical and fumed silica based STFs. They investigated the peak stress attained during the impact loading of STF and judged the impact resistance provided by the STF. The transient response of a colloidal silica-STF using SHPB technique was explored by Lim et al. [20] who studied the effect of loading rate on the transition time for particle volume fraction of 0.54 silica suspension. Asija et al. [21], in another study used SHPB technique for high strainrate characterization of STFs. A progressive increase in the impact toughness of STF with the specimen loading rate was observed by them. They also found the peak stress and peak strain attained along with the characteristic transition time of STF. Cao et al. [22] studied the high strainrate dynamic mechanical properties of STF impregnated Kevlar fabrics using SHPB technique. From their study they concluded that an optimal soft body armor is obtained using multilayer Kevlar fabrics impregnated with high volume fraction of STF. Though this paper does not deal with the experimental characterization of the formulated STFs at high strain-rates, but the claims made in this work of attaining high magnitudes of characteristic viscosities can be clearly justified from the above cited literature.
The power law model (Eq. (1)) was used to estimate the characteristic viscosity $\left(\eta_{\text {char }}\right)$ of the STF, corresponding to characteristic shear rate associated with the ballistic impact. The $\eta_{\text {char }}$ is directly related to the resistance offered to the impacting projectile hence is a measure of the ballistic performance of the STF. The characteristic viscosity of each STF was estimated at the lowest characteristic shear rate of $31525.88 \mathrm{~s}^{-1}$, associated with the armor type IIA. The Fig. 6 shows the effectiveness of the DST samples (STF C, STF D and STF G) over the CST samples (STF A, STF B, STF E, STF F and STF H) as the $\eta_{\text {char }}$ values of the former are higher by many orders of magnitude than the latter. Hence the ballistic performance of a discontinuously shear thickening fluid is better than a continuously shear thickening fluid. Correlating the $\eta_{\text {char }}$ values of the STFs in Fig. 6 to their corresponding $k$ and $n$ values in Table 2 shows that $\eta_{\text {char }}$ is a strong function of flow behavior index $(n)$ compared to flow behavior index $(k)$, which is because $\eta_{\text {char }}$ increases directly with $k$ where

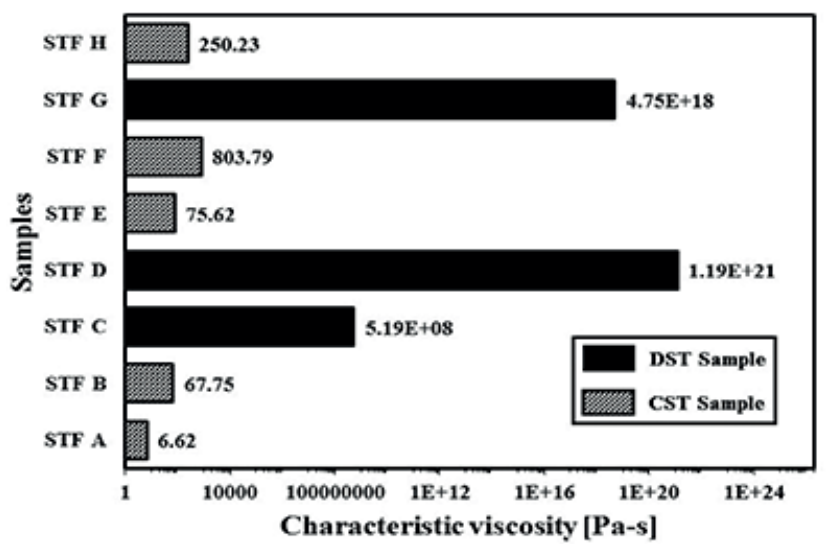

Fig. 6 Comparision of the characteristic viscosities of the STFS (at the characteristics shear rate of $31525.88 \mathrm{~s}^{-1}$ ) 
as exponentially with $n$ (as in Eq. (3)). This led to increase in $\eta_{\text {char }}$ even when $\mathrm{k}$ decreases significantly and $\mathrm{n}$ increases slightly as seen in the cases of increasing $\varphi$ and decreasing $a$. Also, the $\eta_{\text {char }}$ improved with increase in $\eta_{\mathrm{m}}$, where $k$ increased and $n$ remained constant. Consequently, a high $\eta_{\text {char }}$ is favored by high $\varphi$, low $a$, and high $\eta_{\mathrm{m}}$. The power law used here is known to express behavior over a limited range of shear rates, hence may not give realistic extrapolations to high shear rates. Moreover, it was found that the onset of shear thickening was delayed to higher shear rates upon the introduction of a mercury seal at the liquid-air interface in a rheometer setup [15] and when the gap between the rheometer plates is increased [23], indicating that boundary conditions can influence the STF rheology. The boundary conditions of the STF during the rheological testing may not represent the boundary conditions of the STF impregnated in the fabric, hence might lead to a difference between the actual and the assessed performances. Thus, the $\eta_{\text {char }}$ results obtained here may not be accurate, however, are useful to compare the performances of STFs.

$\eta_{\text {char }}=k\left(\dot{\gamma}_{\text {char }}\right)^{n-1}$

\subsection{Design of STF}

The STF for body armor applications, requires that it possess a relatively low pre-transition apparent viscosity (at low shear rates associated with the body movements of the wearer), propensity for rapid transition (upon bullet impact) and a high post-transition viscosity (at high shear rates associated with bullet impact). In terms of rheological properties, the STF with low zero shear viscosity $\left(\eta_{\mathrm{o}}\right)$, low transition time scale and high values of the characteristic viscosity $\left(\eta_{\text {char }}\right)$ gives the best overall performance.

The $\dot{\gamma}_{\text {char }}$ for all the test conditions (Table 3) are three orders of magnitude higher than $\dot{\gamma}_{c}$ values (Table 2) of all the STFs studied here, hence high enough to cause shear thickening in all the samples. However, for the shear thickening effect to be practicable and effective the time scale for the transition should be very low. The time required by STF to make a transition from flowable state to thickened state during the impact event is referred to as the characteristic transition time $\left(t_{\text {char }}\right)$ and it is unique for each STF configuration [19]. In addition to high shear rates, the ballistic events are also associated with high compressive strain rates (order of $10^{5}-10^{7} \mathrm{~s}^{-1}$, [24]). The high strain rate experiments using Split Hopkinson Pressure Bar (SHPB) technique $[19,20,24]$ revealed that the $t_{\text {char }}$ of discontinuously shear thickening fluids (with materials and configurations similar to STFs studied here) is in the order of tens of microseconds at compressive strain rates of order $10^{4} \mathrm{~s}^{-1}$. It was also reported that $t_{\text {char }}$ of discontinuously shear thickening fluids decreases exponentially with compressive strain rates [19, 20] and with particle size of dispersed phase [19]. Thus, a quicker transition is ensured for discontinuously shear thickening fluids with lower particle size, upon ballistic impact. From the previous results (Subsections 3.23.4) we know the favorable STF material configurations to achieve a low $\eta_{\mathrm{o}}$ and high $\eta_{\text {char }}$. Table 4 gives a qualitative summary of the design criteria, the corresponding desirable rheological properties and the favorable material configurations required to achieve them. This provides a general framework to design STF for ballistic applications. It is apparent that material configuration favorable to achieve low pre-transition viscosity leads to decay in the rapidity of transition and the post-transition viscosity. Hence, no conclusion can be drawn about the best material configuration without further insight into the design criteria.

The STF impregnated fabric is stiffer than the neat fabric as the STF introduction causes impedement to the fabric flexure. Thus, the fabric treated with the STF having lower pre-transition apparent viscosity is less stiffer. However, it was reported that the addition of a discontinuously shear thickening fluid (associated with high pre-transition viscosity similar to STF D) to kevlar fabric caused no appreciable increase in its stiffness compared to the neat kevlar [1], indicating that the scope of reducing stiffness of impregnated fabric by lowering the pre-transition viscosity of STF is negligible. Thus, the criterion of low pre-transition viscosity is of little importance and do not carry much weightage in the design of STF. On the other hand, the STF impregnation into kevlar also had enhanced its ballistic resistance such that number of layers of STF treated kevlar required is less than half of that required by neat kevlar to offer equivalent ballistic performance and thereby significantly improving the flexibility [1]. This implies that higher ballistic performance $\left(\eta_{\text {char }}\right)$ of STF gives higher impact resistance for the STF treated fabric

Table 4 Summary of the design criteria, the corresponding desirable rheological properties and the favorable material configurations.

\begin{tabular}{lcc}
\hline Design criteria & $\begin{array}{c}\text { Desirable rheological } \\
\text { property }\end{array}$ & $\begin{array}{c}\text { Favorable material } \\
\text { configuration }\end{array}$ \\
\hline $\begin{array}{l}\text { Low pre-transition } \\
\text { viscosity }\end{array}$ & Low $\eta_{\mathrm{o}}$ & Low $\left(\varphi, \eta_{m}\right), \operatorname{High}(a)$ \\
$\begin{array}{l}\text { Rapid transition } \\
\text { High post-transition } \\
\text { viscosity }\end{array}$ & Low $t_{\text {char }}$ & Low $(a), \operatorname{High}(\varphi)$ \\
\hline
\end{tabular}


and hence a less bulky and more flexible armor. Thus, a high post-transition viscosity and a rapid transition are necessary and sufficient criteria for the STF design. And since the favorable material configuration for these two criteria match (Table 4), the STFs with high $\varphi$ and low $a$ are desirable for ballistic applications.

The packing fraction above which the nature of shear thickening changes from continuous to discontinuous is the critical packing fraction $\left(\varphi_{\text {cd }}\right)$ [15]. Brown and Jaeger [24] have given a practical way to distinguish CST and DST based on flow behavior index $(n)$, according to which STFs were classified as discontinuous if $n>2$ and continuous if $1<n \leq 2$. The $\mathrm{n}$ values obtained for all the STFs (Table 2) are in good agreement with this quantitative classification. They also have suggested that there is no sharp transition between continuous and discontinuous shear thickening such that the former usually evolves into the latter as the packing fraction is increased. This clearly indicates that $\mathrm{n}$ increases continuously with packing fraction and that $\varphi_{\text {cd }}$ corresponds to $n=2$. The $\varphi_{\text {cd }}$ was estimated by interpolating the existing data points of $\varphi$ and $n$ for both the particle sizes (Fig. 7). The value of $\varphi_{\text {cd }}$ is found to be 0.4668 for $400 \mathrm{~nm}$ particle size and 0.4879 for $500 \mathrm{~nm}$ particle size. It can be observed from Fig. 7 that the increase in the severity of shear thickening (n) with packing fraction is pronounced above $\varphi_{\mathrm{cd}}$ for both the $400 \mathrm{~nm}$ and $500 \mathrm{~nm}$ based STFs. It can also be interpreted that for a given packing fraction, shear thickening in smaller particle size based STFs is more severe (high $n$ ) than the large particle based STFs, hence better ballistic performance or high $\eta_{\text {char }}$. Conversely, for a given ballistic performance requirement (fixed $n$ ), considerable weight savings and hence cost reduction (owing to high cost of silica microspheres) can be achieved by using smaller particle size.

Therefore, the use smaller particle size dispersed phase in the STF formulation not only gives quicker transition but also is economical. Furthermore, the key role of the dispersion medium is to transmit stress between the particles as well as between particles and fibres, across the STF impregnated fabric structure. Thus, it is essential that the medium remains in a liquid state to allow for the hydrodynamic interaction of particles and hence shear thickening to occur during impact. The relatively high freezing point of PEG400 $\left(4-8{ }^{\circ} \mathrm{C}\right)$ renders the PEG400 based STFs inutile at sub-freezing temperatures whereas the STFs based on a PEG200 (with freezing point of $-65^{\circ} \mathrm{C}$ ) are operable. Generally for a given polymer, decreasing

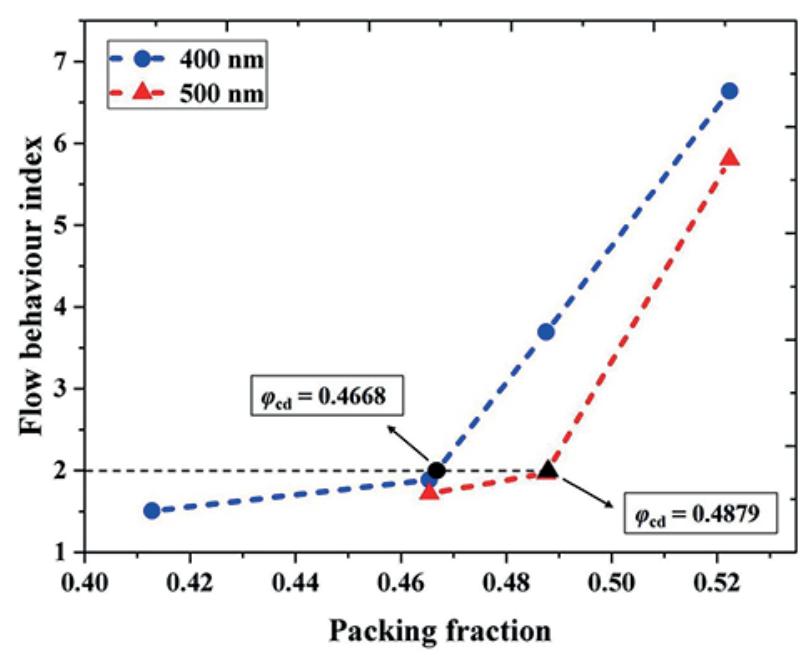

Fig. 7 Effect of packing fraction on the flow behavior index of the STFs with $400 \mathrm{~nm}$ and $500 \mathrm{~nm}$ silica dispersed in PEG200.

degree of polymerization (molecular weight) correlates with lower freezing temperature. Therefore, a lower molecular weight polymeric dispersion medium is favorable as it allows for broader working temperature range of the STF. From the above discussion it can be concluded that the combination of a high packing fraction $\left(\varphi>\varphi_{\text {cd }}\right)$, a low particle size and a low molecular weight dispersion medium is the best STF configuration for ballistic applications. Consequently, the STF D (with $400 \mathrm{~nm}$ silica at 0.5224 packing fraction dispersed in PEG200) is the best choice among the STFs considered for this study.

\subsection{Practical issues}

A marked time dependence was found to accompany shear thickening in stable dispersions [25]. The time dependence refers to the thixotropic and the anti-thixotropic effects. These effects, although practically cannot be completely eliminated, but should limited in magnitude which otherwise affects the reliability of the STF (and hence of the STF-Armor) over time. Also, temperature has a strong effect on the shear thickening behavior. While the critical shear rate decreases, the zero shear viscosity and the severity of shear thickening increases with decrease in temperature [9]. This implies that the performance of the STF impregnated fabric depends on the external temperature. For instance, the STF impregnated ballistic vest developed for moderately hot weather conditions might not be suitable for extremely cold temperatures which a soldier might encounter. Consequently, unlike the conventional soft body armor, a single STFArmor may not be compatible for all weather conditions. 
STFs are sensitive to their environment, as the carrier fluid, glycol, either evaporates or picks up humidity. This will cause changes in the concentration of STF and hence in its rheological properties. In consequence, these materials can find practical use only if they are properly encapsulated [26, 27]. One of the widely adopted ways to enhance the ballistic performance and stab resistance of high strength materials is by impregnating them with STF which poses the problem of STF containment. Soutrenon and Michaud [27] have developed a method in which STFimpregnated foam is encapsulated with silicone, ensuring that the dispersion does not evaporate or gain humidity during service. However, the performance of the soft body armor depends on the load transfer between the layers of the fabric and introduction of foam into the fabric structure may compromise the overall mechanical integrity hence the effectivity of the load transfer. Also the foam makes the armor bulky. Hence, STF-Foam may not be much effective for the ballistic applications. Recently, Asija et al. [28], showed that keeping STF treated ballistic fabrics between untreated layers is an efficient method of STF intercalation compared to keeping the high strength material pouches containing STF between two layers of ballistic materials. This work has introduced a new method that improved the efficacy of STF-treatment however, did not address and resolve the issue of STF containment. Thus, new techniques of manufacture which can effectively incorporate STF into the fabric and enhance the mechanical properties without compromising durability are needed for the successful practical application of the STF-Armor.

\section{Conclusion and future work}

A general framework to design the STF for ballistic applications was constructed by investigating the effects of packing fraction, particle size and continuous phase

\section{References}

[1] Lee, Y. S., Wetzel, E. D., Wagner, N. J. "The ballistic impact characteristics of Kevlar woven fabrics impregnated with a colloidal shear thickening fluid", Journal of Materials Science, 38(13), pp. 2825-2833, 2003. https://doi.org/10.1023/A:1024424200221

[2] Decker, M. J., Halbach, C. J., Nam, C. H., Wagner, N. J., Wetzel, E. D. "Stab resistance of shear thickening fluid (STF)-treated fabrics", Composites Science and Technology, 67(3-4), pp. 565-578, 2007. https://doi.org/10.1016/j.compscitech.2006.08.007

[3] Helber, R., Doncker, F., Bung, R. "Vibration attenuation by passive stiffness switching mounts", Journal of Sound and Vibration, 138(1), pp. 47-57, 1990.

https://doi.org/10.1016/0022-460X(90)90703-3 viscosity on the rheological properties and correlating them with the design criteria. The STFs which thicken discontinuously were shown to be highly effective for impact resistance enhancement compared to the continuous shear thickening fluids. The critical packing fraction $\left(\varphi_{c d}\right)$ for discontinuous shear thickening was estimated to be 0.4668 for $400 \mathrm{~nm}$ particle size and 0.4879 for $500 \mathrm{~nm}$ particle size for a Silica-PEG200 STF. The use smaller particle size dispersed phase in the STF formulation, which is known to give quicker transition was found to be economical as well. A lower molecular weight dispersion medium allows for broader operating temperature range of the STF, hence is favorable. From this study, it was concluded that a high packing fraction $\left(\varphi>\varphi_{\text {cd }}\right)$, a low particle size and a low molecular weight dispersion medium constitute the best STF configuration for ballistic applications. Thus, the STF D, with $400 \mathrm{~nm}$ silica at 0.5224 packing fraction dispersed in PEG200, is the best choice among the STFs considered here. The STF treated fabric composites are less bulky and more flexible substitutes to the conventional soft body armor, therefore, offer a potential solution to extremity protection. Albeit, there are certain limitations and challenges in the development and application of the STF-Armor.

The non-spherical particle based STFs were reported to show shear thickening intensities similar to the STFs composed of spherical particles at much lower packing fractions, hence are better alternatives. The effect of particle shape on the STF design characteristics will be studied comprehensively in the future.

\section{Acknowledgement}

This work was supported by the Terminal Ballistics Research Laboratory (TBRL) of Defence Research and Development Organization (DRDO).

[4] Laun, H. M. "Rheology of extremely shear thickening polymer dispersions $^{\text {a) }}$ (passively viscosity switching fluids)", Journal of Rheology, 35(6), pp. 999, 1991. https://doi.org/10.1122/1.550257

[5] Fischer, C., Braun, S. A., Bourban, P.-E., Michaud, V., Plummer, C. J. G., Månson, J. E., Manson, J.-A. E. "Dynamic properties of sandwich structures with integrated shear-thickening fluids", Smart Materials and Structures, 15(5), pp. 1467-1475, 2006. https://doi.org/10.1088/0964-1726/15/5/036

[6] Zhang, X. Z., Li, W. H., Gong, X. L. "The rheology of shear thickening fluid (STF) and the dynamic performance of an STF-filled damper", Smart Materials and Structures, 17(3), ID. 035027, 2008. https://doi.org/10.1088/0964-1726/17/3/035027 
[7] Rabb, R. J., Fahrenthold, E. P. "Evaluation of Shear-ThickeningFluid Kevlar for Large-Fragment-Containment Applications", Journal of Aircraft, 48(1), pp. 230-234, 2011. https://doi.org/10.2514/1.C031081

[8] Warren, J., Kota, K. R., Westberg, S. M., Lacy, T., Kundu, S., Toghiani, H., Pittman, C. U. "Hypervelocity impacts of shear thickening fluid imbibed metallic foam core sandwich panels", presented at 30th technical conference of American Society of Composites, East Lansing, USA, Sept. 28-30, 2015.

[9] Warren, J., Offenberger, S., Toghiani, H., Pittman, C. U., Lacy, T. E., Kundu, S. "Effect of Temperature on the Shear-Thickening Behavior of Fumed Silica Suspensions", ACS Applied Materials and Interfaces, 7(33), pp. 18650-18661, 2015.

https://doi.org/10.1021/acsami.5b05094

[10] Srivastava, A., Majumdar, A., Butola, B. S. "Improving the Impact Resistance of Textile Structures by using Shear Thickening Fluids: A Review", Critical Reviews in Solid State and Materials Sciences, 37(2), pp. 115-129, 2012. https://doi.org/10.1080/10408436.2011.613493

[11] Jacobs, M. J. N., Van Dingenen, J. L. J. "Ballistic protection mechanisms in personal armour", Journal of Materials Science, 36(13), pp. 3137-3142, 2001.

https://doi.org/10.1023/A:1017922000090

[12] Wagner, N. J., Lee, Y. S., Kirkwood, K. "Advanced Body Armor Utilizing Shear Thickening Fluids", presented at 23rd Army Science Conference, Orlando, Florida, USA, Dec. 2-5, 2002.

[13] Hoffman, R. L. "Discontinuous and Dilatant Viscosity Behavior in Concentrated Suspensions, Part II. Theory and Experimental Tests", Journal of Colloid and Interface Science, 46(3), pp. 491-506, 1974. https://doi.org/10.1016/0021-9797(74)90059-9

[14] Brady, J. "Stokesian Dynamics", Annual Review of Fluid Mechanics, 20(1), pp. 111-157, 1988. https://doi.org/10.1146/annurev.fluid.20.1.111

[15] O’Brien, V. T., Mackay, M. E. "Stress components and shear thickening of concentrated hard sphere suspensions", Langmuir, 16(21), pp. 7931-7938, 2000. https://doi.org/10.1021/la000050h

[16] Dodge, D., Metzner, A. "Turbulent Flow of Non-Newtonian Systems", AIChE Journal, 5(2), pp. 189-203, 1959. https://doi.org/10.1002/aic.690050214

[17] Barnes, H. A. "Shear-Thickening ("Dilatancy") in Suspensions of Nonaggregating Solid Particles Dispersed in Newtonian Liquids", Journal of Rheology, 33(2), pp. 329-366, 1989. https://doi.org/10.1122/1.550017

[18] U.S. Department of Justice, Office of Justice Programs, National Institute of Justice "NIJ Standard-0101.06, 2008, Ballistic resistance of personal body armor", National Institute of Justice, USA, 2008.
[19] Asija, N., Chouhan, H., Gebremeskel, S. A., Bhatnagar, N. "Influence of particle size on the low and high strain rate behavior of dense colloidal dispersions of nanosilica", Journal of Nanoparticle Research, 19(1), pp. 21, 2017. https://doi.org/10.1007/s11051-016-3723-4

[20] Lim, A. S., Lopatnikov, S. L., Wagner, N. J., Gillespie, J. W. "Investigating the transient response of a shear thickening fluid using the split Hopkinson pressure bar technique", Rheologica Acta, 49(8), pp. 879-890, 2010. https://doi.org/10.1007/s00397-010-0463-8

[21] Asija, N., Chouhan, H., Gebremeskel, S. A., Bhatnagar, N. "High strain rate characterization of shear thickening fluids using Split Hopkinson Pressure Bar technique", International Journal of Impact Engineering, 110, pp. 365-370, 2017. https://doi.org/10.1016/j.ijimpeng.2017.02.018

[22] Cao, S., Chen, Q., Wang, Y., Xuan, S., Jiang, W., Gong, X. "High strain-rate dynamic mechanical properties of Kevlar fabrics impregnated with shear thickening fluid", Composites Part A: Applied Science and Manufacturing, 100, pp. 161-169, 2017. https://doi.org/10.1016/j.compositesa.2017.04.015

[23] Fall, A., Huang, N., Bertrand, F., Ovarlez, G., Bonn, D. "Shear Thickening of Cornstarch Suspensions as a Reentrant Jamming Transition", Physical Review Letters, ID 018301, 2008. https://doi.org/10.1103/PhysRevLett.100.018301

[24] Brown, E., Jaeger, H. M. "Shear thickening in concentrated suspensions: Phenomenology, mechanisms and relations to jamming", Reports on Progress in Physics, 77(046602), pp. 1-23, 2014. https://doi.org/10.1088/0034-4885/77/4/046602

[25] Boersma, W. H. "Time-dependent behavior and wall slip in concentrated shear thickening dispersions", Journal of Rheology, 35(6), pp. 1093, 1991. https://doi.org/10.1122/1.550167

[26] Soutrenon, M., Michaud, V., Manson, J. E. "Influence of processing and storage on the shear thickening properties of highly concentrated monodisperse silica particles in polyethylene glycol", Applied Rheology, 23(5), pp. 287-295, 2013. https://doi.org/10.3933/ApplRheol-23-54865

[27] Soutrenon, M., Michaud, V. "Impact properties of shear thickening fluid impregnated foams", Smart Materials and Structures, 23(035022), pp. 1-10, 2014. https://doi.org/10.1088/0964-1726/23/3/035022

[28] Asija, N., Chouhan, H., Gebremeskel, S. A., Bhatnagar, N. "Impact Response of Shear Thickening Fluid (STF) Treated High Strength Polymer Composites - Effect of STF Intercalation Method", Procedia Engineering, 173, pp. 655-662, 2017. https://doi.org/10.1016/j.proeng.2016.12.133 